\title{
Iris Recognition based on Wavelets
}

\author{
S V Sheela \\ B M S College of \\ Engineering \\ Bangalore, India.
}

\author{
P A Vijaya \\ Malnad College of Engineering \\ Hassan, India
}

\begin{abstract}
Recognition refers to the problem of establishing a subject's identity from a set of already known identities. Iris recognition system identifies a person from the database of iris images. Iris patterns form distinguishing characteristics for an individual. The potency of iris recognition lies in its textual information. Iris based security systems capture iris patterns of individuals and match the patterns against the record in available databases. In this paper, wavelet decomposition is applied on iris patterns. The magnitude of coefficients aid in the generation of unique code for recognition. The recognition rate of $100 \%$ is achieved.
\end{abstract}

\section{Keywords}

Wavelet decomposition, unique code, magnitude of detailed coefficients, core and non-core segments

\section{INTRODUCTION}

Biometric is a metric of apparent nontransferable uniqueness provided by user's presence. Biometrics are extremely convenient form of providing identity and cannot

be lent to another individual. The digital impersonation provides usage of biometrics in security techniques. A measurable physical characteristic is more reliable to eliminate identity theft. Iris biometry is used to recognize an individual in a natural and intuitive way. Iris is the annular ring between pupil and sclera of eye. The sclera is the white region of connective tissues surrounding iris. The pupil is the darkest region in eye. The iris is highly protected and non-invasive. The structure of iris exhibits long-term stability. The texture of the iris presents distinguishing characteristics. The variations in the gray level intensity values distinguish two individuals. The attributes important for largescale identity programs are accuracy, algorithm speed and template size. The security systems, database authentication systems and secure transactions are major applications. The iris recognition algorithms need to be developed and tested in diverse environment and configurations. Research issues are based on iris localization, normalization, occlusion, segmentation,

liveness detection and handling noisy and degraded iris images. The occlusion is due to eyelid and eyelashes. Iris liveness detection differentiates live subject from a photograph, a video playback, a glass eye or other artifacts. Biometric security techniques look for unique physiological characteristics that remain constant and difficult to fake. A blend of acquisition, feature extraction and classification has evidently become technology oriented in e-commerce and m-commerce applications. The most common features are $x-y$ coordinates of pupil, intensity value, radius of pupil, radius of iris, eye corners and ratio of limbus diameter to pupil diameter. The derived features are intensity gradient, phase information, texture difference, zero crossings, moment values, wavelet decomposition coefficients, local intensity variations, independent component analysis, hierarchical texture information, fractal dimension, zerotree wavelet code, local-global graphs and mean/standard deviation of image. The general classifiers used are support vector machine, radial basis function, genetic algorithm, component analysis, $k$-means, fuzzy $k$-means, $k$-nearest neighbor and neural networks. The metrics used to identify an individual are sequence codes such as iriscode.

\section{PROLOGUE \\ 2.1 Connected Components Labeling}

The Connectivity between pixels of gray scale images is determined based on gray level intensity values and spatial adjacency [1]. The neighborhood system $N S_{d}$ is defined for $d=4$, 8. Two pixels $p_{1}, p_{2} \in N S_{4}$ are called 4-adjacent, if they are vertical or horizontal neighbors. The pixels $p_{1}, p_{2} \in N S_{8}$ are called 8 -adjacent, if they are vertical, horizontal or diagonal neighbors. A set of pixels $C$ is d-connected if for every pair of pixels $c_{i}, c_{j} \in C$, there exists a sequence of pixels $c_{i}, \ldots, c_{j}$ such that (i) all pixels in the sequence share same intensity value and (ii) each consecutive pair in the sequence is d-adjacent [3]. The region of connected pixels is called connected component [4]. The distinct regions in an image are identified.

Connected component labeling assigns a label to each pixel representing the region to which it belongs. The labeling process scans the image in horizontal and vertical directions for white pixels. Each unlabeled pixel $I(x, y)$ is considered. The 8-connected neighbors, $(m, n)$ are listed. The criteria is based on the presence of same intensity value in the neighboring pixels. A label is assigned in label matrix, $M_{\text {label }}$ corresponding to position $(x, y)$, if $I(m, n)$ is equal to $I(x, y)$. The method labels all pixels in the 8connected region to which pixel belongs [4]. The sequence of steps involved in labeling process is shown in Figures 1(a)-(e). The connected components are labeled using (1).

$$
\left[M_{\text {label }}, n\right]=\operatorname{com}_{\text {label }}(B)
$$

where $n$ is the number of connected components and $B$ is the binary image. 


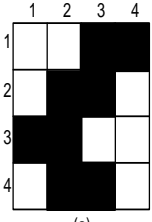

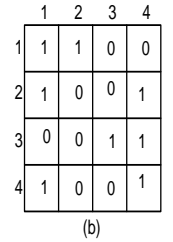
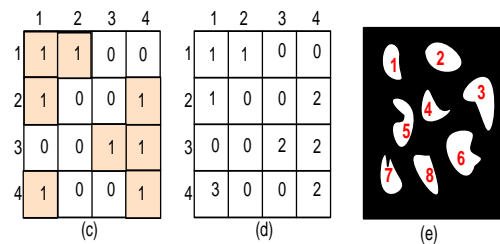

Fig 1. (a) Binary image (b) Intensity values (c) Connected components (d) Label matrix (e) An instance of component labelling

The area of all white pixels is summation of individual pixel areas in the image. In a $2 \times 2$ neighborhood, considering only the white pixels, the area of one pixel is 0.25 , two adjacent pixels is 0.5 , two diagonal pixels is 0.75 , three pixels is 0.875 and four pixels is 1 . The area is determined using (2) for each connected component.

$$
\begin{gathered}
C(k)=\operatorname{com}_{\text {area }}(k), k=1,2, \ldots n \text { and } M_{\text {label }}=k \\
m_{\text {com }}=\max (C)
\end{gathered}
$$

The vector $C$ consists of component areas. The component with maximum area is determined using (3).

\subsection{Discrete Wavelet Transform}

Wavelet transform provides the time-frequency representation. In case of discrete wavelet transform (DWT), filters of different cutoff frequencies are used to analyze the signal at different scales [5]. Wavelets are mathematical functions that divides the data into different frequency components to analyze each component with a resolution matched to its scale [6]. Wavelet decomposition involves a pair of waveforms, the wavelet function and the scaling function. The wavelet function represents high frequencies corresponding to the detailed parts of an image. The scaling function represents the low frequencies or smooth parts of an image. The DWT analyzes the signal by decomposing the signal into a coarse approximation and detail information. The scaled and translated basis functions in 2D DWT is given by (4) and (5).

$$
\begin{aligned}
& \phi_{k, p, q}(x, y)=2^{k / 2} \varphi\left(2^{k} x-p, 2^{k} y-q\right) \\
& \psi_{k, p, q}^{i}(x, y)=2^{k / 2} \psi^{i}\left(2^{k} x-p, 2^{k} y-q\right)
\end{aligned}
$$

where $i$ is the index to identify the directional wavelets in terms of horizontal, vertical and diagonal components.

In two level decomposition, the upper left quadrant is the approximation of the source image. The upper right, lower left and lower right represent vertical, horizontal and diagonal details of the source. The approximation at level 1 is decomposed into four components at level 2. The wavelet transform outputs at each level the approximation, horizontal detail, vertical detail and diagonal detail. The size of each subimage is reduced to quarter of the source image.

The Haar basis is obtained with a multiresolution of piecewise constant functions. The scaling function is $\phi=1_{[0,1]}$. The wavelet function is given by $\psi(t)=-1$ if $0 \leq t \leq 0.5,1$ if $0.5 \leq t \leq 1,0$ otherwise. It is the only symmetric wavelet with the advantages of being fast and memory efficient. Haar wavelets is conceptually simple, but not suitable for approximating smoothing functions that is required in applications like compression. Haar wavelet has only one vanishing moment. The vanishing moment of $v$ indicates that any polynomial signal up to order $v-1$ is represented completely in scaling space.

Daubechies family of scale functions have finite vanishing moments. This property is useful for local analysis and insures the number of non-zero coefficients in the associated filter to be finite [7]. The scaling function is given by $\phi(x)=\sum_{i=0}^{N-1} p_{i} \phi(2 x-i)$ and wavelet function is $\psi(x)=\sum_{i=2-N}^{1}(-1)^{i} p_{1-i} \phi(2 x-i)$ where $i=1,2, \ldots, N-1$ are the filter coefficients [8]. Daubechies wavelets are asymmetric. By optimizing the real coefficients $R\left(e^{i w}\right)$ of the minimum degree polynomial $P\left(\left(2-e^{i w}-e^{-i w}\right) / 4\right)$, the linear phase and symmetric property is obtained. Thesymmetric wavelets are the symlets. Coiflet family of wavelets have $v$ vanishing moments. The scaling functions are based on the constraints, $\int \phi(t) d t=1$ and $\int p^{k} \phi(p) d p=0$ for $1 \leq k \leq v$. The coiflets are useful for establishing precise quadrature formulas. Biorthogonal wavelets are families of compactly supported symmetric wavelets. The symmetry of the filter coefficients provides linear phase of the transfer function [7]. Two scaling functions, $\phi, \tilde{\phi}$ and two wavelet functions, $\psi, \tilde{\psi}$ are defined such that $\int \psi_{j, k}(x) \tilde{\psi}_{j^{\prime}, k^{\prime}}(x) d x=0$ for $j \neq j^{\prime}$ or $k \neq k^{\prime}$ and $\int \phi_{0, k}(x) \tilde{\phi}_{0, k^{\prime}}(x) d x=0$ for $k \neq k^{\prime}$. The properties of analysis are concentrated on $\tilde{\psi}$ function and synthesis on $\psi$ function.

\section{RELATED WORK}

The iris recognition system by J.Daugman use phase-based approach 10 . The representation of iris texture is binary coded by quantizing the phase response of a texture filter using quadrature 2D Gabor wavelets into four levels. Iris codes are generated and Hamming Distance is used as a measure of dissimilarity. Continuing the Daugman's method, Karen Hollingsworth has developed techniques for

improving recognition rates [10]. The techniques include fragile bit masking, signal level fusion of iris images, detecting local distortions in iris texture and analyzing the effects of pupil dilation. The experiments are conducted on ICE database. The system developed by Wildes is based on texture analysis [11]. The Laplacian of Gaussian (LoG) is applied to the image at multiple scales and the resulting Laplacian pyramid constructed with different levels serve as basis for further processing. Iris recognition system developed by $\mathrm{Li} \mathrm{Ma}$ is characterized by local intensity variations [12]. The sharp variation points of iris patterns are recorded as features. The feature extraction generates 1D intensity signals considering the information density in the angular direction. The feature values are the mean and the average absolute deviation of the magnitude of each $8 \times 8$ block in the filtered image. The method by $\mathrm{Li} \mathrm{Ma}$ was further improved by Zhenan Sun where in the local feature based classifier was combined with an iris blob matcher [13]. The blob matching aimed at finding the spatial correspondences between the blocks in the input image and that in the stored model. The similarity is 
based on the number of matched block pairs. The block attributes are recorded as centroid coordinates, area and second order central moments. H. Proenca proposed a moment-based texture segmentation algorithm, using second order geometric moments of the image as texture features [14]. The iris recognition system by Aditya Abhyankar is based on biorthogonal 5/3 tap wavelets [15]. The experiment is conducted on CASIAv1 database. The match-score calculation was performed using Hamming Distance and FRR of $4.2 \%$ is obtained with a threshold of 0.25 . The method by Agus Harjoko use 1D coiflet wavelet for iris recognition [16]. Coiflet wavelet transform is applied to the iris image from CASIAv1 database. The second order wavelets was implemented with four decomposition levels.

Hamming Distance is used as decision criteria and a success rate of $84.25 \%$ was obtained.

\section{PROPOSED METHOD}

\subsection{Preprocessing}

The $640 \times 480$ sized eye image $I$ is shown in Figure 2(a). The histogram $H$, of $I$ provides the count of pixels $p c$ for each intensity value using (6). The first peak in the histogram is the threshold value $h$ computed using (7). The intensity values less than or equal to $h$ are obtained as a intermediate binary image $B$ using (8). The white pixels in $B$ corresponds to pupil and eyelashes as shown in Figure 2(b). The intensity values greater than $h$ is the sclera and eyelids.

$$
\begin{aligned}
& p c=H(I(x, y)) \\
& h=\max (p c) \\
& B=I(x, y)<=h
\end{aligned}
$$

The result of determining maximum area of connected components is a binary image such that pupil area is white and other parts of image are black as shown in Figure 2(c). The maximum and minimum values of $\mathrm{x}$-coordinates corresponding to the white pixels, $x_{\max }, x_{\min }$ are identified. Similarly, the maximum and minimum values of $y$-coordinates corresponding to white pixels, $y_{\max }, y_{\min }$ are determined. The centre of pupil is calculated using $x_{c}=\left(x_{\max }+x_{\min }\right) / 2$ and $y_{c}=\left(y_{\max }+y_{\min }\right) / 2$. The radius of both axes is determined using $\operatorname{rad}_{1}=\left(x_{\max }-x_{\min }\right) / 2$ and $\mathrm{rad}_{2}=$ $\left(y_{\max }-y_{\min }\right) / 2$. The pupil radius, $\operatorname{rad}_{p}=\max \left(\operatorname{rad}_{1}, \operatorname{rad}_{2}\right)$. A mapping is achieved to original gray scale image using coordinates of pupil center and radius.

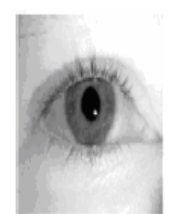

(a)

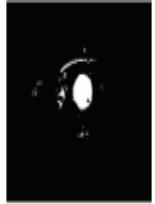

(b)

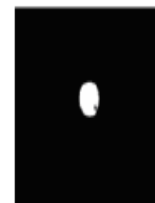

(c)

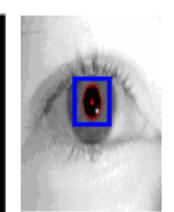

(d)

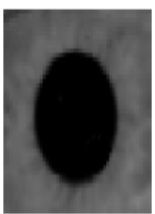

(e)
Fig. 2 (a) Eye image (b) Connected components (c) Component with maximum area (d) Pupil and iris detection (e) Segmented image

A normalised bounding box is defined to extract the segmented image based on the pupil detection as shown in Figure 2(d). The segmented image shown in Figure 2(e) consists iris portion surrounding the pupil. The texture in this part of image show maximum randomness to form unique patterns.

\subsection{Wavelet Decomposition}

An image $I$ of size $\mathrm{MxN}$ is considered. The one-dimensional DWT applied to each row produces two MxN/2 images. Further, DWT is applied column-wise on these two images to obtain four $\mathrm{M} / 2 \mathrm{xN} / 2$ images. The approximation component, app comp represents the coarser information of the input image. The wavelets measure gray level variations in three orientations. The detail information is contained in the horizontal, vertical and diagonal coefficients denoted as hor comp, ver $_{\text {comp }}$ and dia comp. The variations along columns represent the horizontal details, the variations along rows gives the vertical details and the variations in the diagonal direction gives the diagonal coefficients [17]. DWT was applied for the non-core segments, ncseg $_{i}$ and core segments, cseg $_{j}$ independently, where $i \in\{1,2,3,4,5,8,9,11$, $12,13,14,15,16\}$ and $j \in\{6,7,10,11\}$. The core and non-core segments are shown in Figure 3.

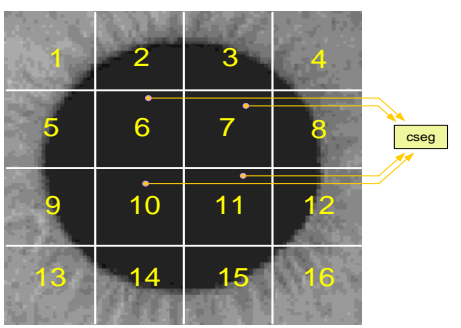

Fig. 3: Segments in iris image

A single level discrete 2-D wavelet transform was applied. The types of wavelet families used are Haar, Daubechies, Symlets, Coiflets and Biorthogonal. The 2D DWT leads to a decomposition of approximation coefficients at level $j$ into four components the approximation $a p p_{c o m p}$ at level $j+1$, and the details in three orientations, horizontal hor comp$_{\text {, vertical }}$ ver $_{\text {comp, }}$, and diagonal dia $_{\text {comp. }}$. The decomposition

is given by (9).

$\left(a p p_{\text {comp }}\right.$, hor $_{\text {comp }}, v_{\text {eromp }}$, dia $\left._{\text {comp }}\right)=$ wave_trans $($ img $)$

where img corresponds to cseg and ncseg of the image.

The hor ${ }_{\text {comp }}$, ver ${ }_{\text {comp }}$ and dia $_{\text {comp }}$ coefficient values by applying db4 wavelet transform for each segment are tabulated in Table 1. The coefficient values in core segments of all samples are similar. The values are constant due to the texture of the pupil. The intensity values in this region is less or nearing zero. The coefficient values of cseg are eliminated. The values obtained from ncseg is significant and used for further processing.

Table 1. Detail coefficient values

\begin{tabular}{|l|l|l|l|}
\hline segment & hor $_{\text {comp }}$ & ver $_{\text {comp }}$ & dia $_{\text {comp }}$ \\
\hline ncseg $_{1}$ & 0.0765 & 0.1980 & -0.0214 \\
\hline ncseg $_{2}$ & 0.0958 & 0.0623 & -0.0145 \\
\hline ncseg $_{3}$ & -0.0346 & -0.0577 & 0.0141 \\
\hline ncseg $_{4}$ & 0.0327 & -0.0870 & 0.0062 \\
\hline ncseg $_{5}$ & 0.0328 & 0.1924 & 0.0049 \\
\hline ncseg $_{8}$ & 0.0664 & 0.1454 & 0.0124 \\
\hline ncseg $_{9}$ & 0.0457 & 0.2724 & -0.0031 \\
\hline ncseg $_{12}$ & 0.0661 & 0.1548 & 0.00099 \\
\hline
\end{tabular}




\begin{tabular}{|l|l|l|l|}
\hline ncseg $_{13}$ & 0.0231 & -0.0764 & 0.0165 \\
\hline ncseg $_{14}$ & 0.1506 & -0.0594 & -0.0076 \\
\hline ncseg $_{15}$ & 0.1891 & -0.0746 & -0.0047 \\
\hline ncseg $_{16}$ & -0.1164 & -0.1989 & 0.0223 \\
\hline $\operatorname{cseg}_{6}$ & $-5.3291 \mathrm{e}-015$ & $2.5121 \mathrm{e}-015$ & $-4.9304 \mathrm{e}-032$ \\
\hline $\operatorname{cseg}_{7}$ & $-5.3291 \mathrm{e}-015$ & $2.5121 \mathrm{e}-015$ & $-4.9304 \mathrm{e}-032$ \\
\hline $\operatorname{cseg}_{10}$ & $-5.3291 \mathrm{e}-015$ & $2.5121 \mathrm{e}-015$ & $-4.9304 \mathrm{e}-032$ \\
\hline $\operatorname{cseg}_{11}$ & $-5.3291 \mathrm{e}-015$ & $2.5121 \mathrm{e}-015$ & $-4.9304 \mathrm{e}-032$ \\
\hline
\end{tabular}

The approximation and the detail coefficients for $n c s e g_{8}$ of genuine and imposter samples are shown in Figures 4(a)-(d).

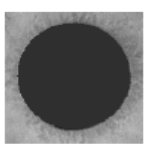

(a)

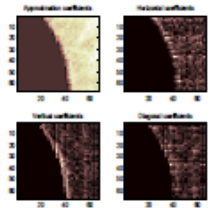

(b)

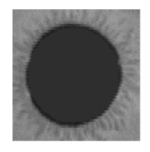

(c)

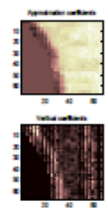

(d)
Fig. 4 (a) Genuine sample (b) db4 wavelet transform applied to ncseg $_{8}$ of genuine sample (c) imposter sample (d) db4 wavelet transform applied to ncseg $_{8}$ of imposter sample

It is observed that coefficient values in each segment of genuine and imposter samples are different. The difference is mainly due to the variations in the texture of the iris. The part of the iris close to the pupil show variations between genuine and imposter samples. The wavelet types, Haar, symlets and coiflets are applied on non-segments. The approximation and detail coefficients for ncseg $_{8}$ are shown in Figures 5(a)-(c).

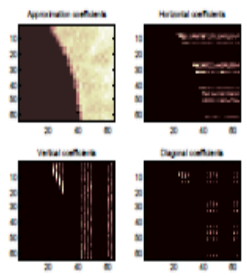

(a)

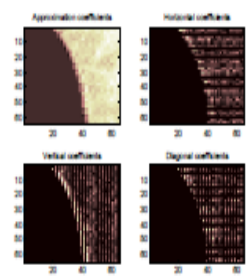

(b)

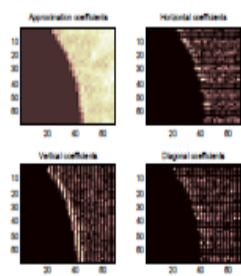

(c)
Fig. 5: (a) Haar wavelet transform (b) Symlets transform (c) Coiflets transform

The coefficient values of ncseg are used in summation and magnitude based approaches.

\section{SUMMATION BASED APPROACH}

The mean values of the horizontal, vertical and diagonal coefficients are denoted by mhor, mver and mdia. The summation of mean values are computed for ncseg and cseg using (10) and (11).

$$
\begin{aligned}
& n c s_{\text {hor }}=\sum_{i} \operatorname{mhor}\left(\text { ncseg }_{i}\right) \\
& n c s_{\text {ver }}=\sum_{i} \operatorname{mver}\left(\text { ncseg }_{i}\right) \\
& n c s_{d i a}=\sum_{i} \operatorname{mdia}\left(\text { ncseg }_{i}\right)
\end{aligned}
$$

where $i=\{1,2,3,4,5,8,9,12,13,14,15\}$.

$$
\begin{aligned}
& c s_{\text {hor }}=\sum_{j} \operatorname{mhor}\left(\operatorname{cseg}_{j}\right) \\
& c s_{v e r}=\sum_{j} \operatorname{mver}\left(\operatorname{cseg}_{j}\right) \\
& c s_{d i a}=\sum_{j} \operatorname{mdia}\left(\operatorname{cseg}_{j}\right)
\end{aligned}
$$

where $j=\{6,7,10,11\}$.

The cumulative difference, ratio, z-norm and Pearson correlation coefficient of the summation values along the three orientations are computed. The cumulative difference of the summation values along the three orientations are computed using (12).

$$
\begin{aligned}
& \operatorname{diff}_{n c s}=n s_{d i a}-n s_{v e r}-n s_{h o r} \\
& \operatorname{diff}_{c s}=c s_{d i a}-c s_{v e r}-c s_{h o r}
\end{aligned}
$$

Ratio of differences is given by $r$ diff $=$ diff ${ }_{n c s} /$ diff $c$ s. The rdiff values are in different range for genuine and imposter samples. The rsum given by (13) indicates the ratio of the summation values in horizontal and vertical directions of non-core segments with respect to core segments. The values of imposter samples differ from that of the genuine.

$$
r s u m=\left(n c s_{\text {hor }}+n c s_{\text {ver }}\right) /\left(c s_{\text {hor }}+c s_{\text {ver }}\right)
$$

Table 2: Difference and ratio of summation values for genuine samples

\begin{tabular}{|c|c|c|c|c|}
\hline Sample & diff $_{\text {ncs }}$ & diff $_{c s}$ & rdiff & rsum \\
\hline 1 & $-8.32 \mathrm{E}-01$ & $-3.43 \mathrm{E}-14$ & $2.12 \mathrm{E}+13$ & $2.58 \mathrm{E}+13$ \\
\hline 2 & $-8.73 \mathrm{E}-01$ & $-3.83 \mathrm{E}-14$ & $1.95 \mathrm{E}+13$ & $2.03 \mathrm{E}+13$ \\
\hline 3 & $-7.94 \mathrm{E}-01$ & $-3.13 \mathrm{E}-14$ & $2.05 \mathrm{E}+13$ & $2.09 \mathrm{E}+13$ \\
\hline 4 & $-8.10 \mathrm{E}-01$ & $-3.44 \mathrm{E}-14$ & $2.03 \mathrm{E}+13$ & $2.04 \mathrm{E}+13$ \\
\hline
\end{tabular}

Table 3: Difference and ratio of summation values for imposter samples

\begin{tabular}{|c|c|c|c|c|}
\hline Sample & diff $_{n c s}$ & diff & rdiff & rsum \\
\hline 1 & $-2.04 \mathrm{E}+01$ & $-3.72 \mathrm{E}-13$ & $6.05 \mathrm{E}+12$ & $6.42 \mathrm{E}+12$ \\
\hline 2 & $-2.64 \mathrm{E}+01$ & $-3.24 \mathrm{E}-13$ & $5.24 \mathrm{E}+12$ & $5.41 \mathrm{E}+12$ \\
\hline 3 & $-2.11 \mathrm{E}+01$ & $-2.72 \mathrm{E}-13$ & $6.47 \mathrm{E}+12$ & $6.59 \mathrm{E}+12$ \\
\hline 4 & $-2.33 \mathrm{E}+01$ & $-3.14 \mathrm{E}-13$ & $5.26 \mathrm{E}+12$ & $4.36 \mathrm{E}+12$ \\
\hline
\end{tabular}

The summation, $h v_{i}=n c s_{h o r}+n c s_{v e r}+c s_{h o r}+c s_{v e r}$ is calculated where $i=\{1,2,3,4,5,6,7,8,9,10\}$ maps to $\mathrm{wt}_{\text {order. }}$. The $w t_{\text {order }}$ corresponds to the different types of wavelets applied in the order, $\{\mathrm{db} 3, \mathrm{db} 2$, db1, db4, db5, db6, Haar, sym, coif, biof\}.

The successive differences of the summation values, $d h v$ is computed by (14). For instance, the $d h v_{3}$ values represent the difference of $h v$ values for $\mathrm{db} 1$ and $\mathrm{db} 4$ transforms. The values prove divergent for genuine and imposter samples.

$$
d h v_{j}=h v_{j+1}-h v_{j} \text { where } j=\{1,2,3,4,5,6,7,8,9\}
$$

The experiment was continued by estimating successive ratios of the $h v$ values. The successive ratios $r h v$ values are calculated according to $w t_{\text {order }}$. For instance, the $r h v_{3}$ values denotes the ratio of $h v$ values by applying $\mathrm{db} 1$ and $\mathrm{db} 4$ wavelet transform. The $r h v$ 
values for genuine samples are given in Table 4. The $r h v$ values for imposter samples are given in Table 5. The values are different for genuine and

imposter samples.

Table 4. $r h v$ values for genuine samples

\begin{tabular}{|c|c|c|c|c|c|c|c|c|c|}
\hline $\begin{array}{c}\text { Sampl } \\
\mathrm{e}\end{array}$ & $r h v_{1}$ & $r h v$ & $r h v$ & $r h v$ & $r h v$ & $r h v$ & $r h v$ & $r h v$ & $r h v$ \\
2 & 3 & 4 & 5 & 6 & 7 & 8 & 9 \\
\hline 1 & 1.1 & 0.7 & 1.2 & 0.9 & 1.1 & 1.1 & 0.8 & 0.9 & 1.2 \\
\hline 2 & 1.1 & 0.7 & 1.3 & 0.7 & 1.1 & 2.1 & 0.5 & 0.9 & 1.9 \\
\hline 3 & 0.9 & 1.5 & 1.4 & 0.5 & 1.1 & 2.1 & 0.2 & 1.3 & 2.2 \\
\hline 4 & 1.0 & 1.2 & 1.3 & 0.6 & 1.1 & 2.4 & 0.3 & 0.7 & 2.4 \\
\hline 5 & 1.7 & 1.5 & 1.4 & 0.2 & 0.9 & 2.5 & 0.2 & 0.2 & 2.1 \\
\hline 6 & 1.2 & 0.9 & 1.4 & 0.4 & 1.2 & 2.3 & 0.6 & 0.5 & 1.9 \\
\hline 7 & 1.1 & 0.7 & 1.3 & 0.7 & 1.0 & 1.4 & 0.9 & 0.7 & 1.4 \\
\hline
\end{tabular}

Table 5. $r h v$ values for imposter samples

\begin{tabular}{|c|c|c|c|c|c|c|c|c|c|}
\hline Sample & $r h v_{1}$ & $r h v_{2}$ & $r h v_{3}$ & $r h v_{4}$ & $r h v_{5}$ & $r h v_{6}$ & $r h v_{7}$ & $r h v_{8}$ & $r h v_{9}$ \\
\hline 1 & 2.7 & 3.9 & 2.5 & 3.6 & 4.6 & 3.4 & 3.2 & 4.2 & 3.7 \\
\hline 2 & 3.0 & 4.2 & 2.7 & 3.2 & 4.0 & 4.2 & 3.6 & 4.2 & 4.1 \\
\hline 3 & 2.7 & 3.6 & 2.7 & 3.5 & 4.6 & 3.1 & 4.3 & 3.8 & 4.3 \\
\hline 4 & 2.8 & 4.5 & 2.6 & 3.3 & 4.1 & 4.3 & 3.9 & 4.3 & 3.8 \\
\hline 5 & 3.1 & 3.8 & 2.6 & 3.2 & 4.1 & 3.9 & 4.3 & 3.7 & 4.3 \\
\hline 6 & 3.1 & 3.7 & 2.5 & 2.8 & 3.8 & 3.4 & 3.7 & 3.7 & 4.4 \\
\hline 7 & 3.2 & 3.1 & 2.6 & 3.5 & 4.5 & 3.4 & 4.5 & 3.8 & 4.3 \\
\hline
\end{tabular}

The $\mathrm{z}$-score was applied for providing promising results. The $\mathrm{z}-$ score, $\mathrm{Z}_{\mathrm{i}}$ was computed for $h v$ values with $i=\{1 . .10\}$, indicating the type of the wavelet in $\mathrm{wt}_{\text {order }}$. The $\mathrm{z}$-score indicates the deviation of $h v$ values with respect to the mean and in terms of the standard deviation. The z-score is computed as $Z_{i}=(x-\bar{x}) / \sigma$

where $\mathrm{Z}$ is the $\mathrm{z}$-score, $x$ is the $h v$ values, $\bar{x}$ is the mean of the $h v$ values and $\sigma$ is the standard deviation. The plot of $\mathrm{Z}_{4}$ values for genuine and imposter samples is given in Figure 6. It was observed that $\mathrm{z}$-score values of genuine vary from that of an imposter. $\mathrm{Z}_{4}$ corresponds to $\mathrm{db} 4$ wavelet type.

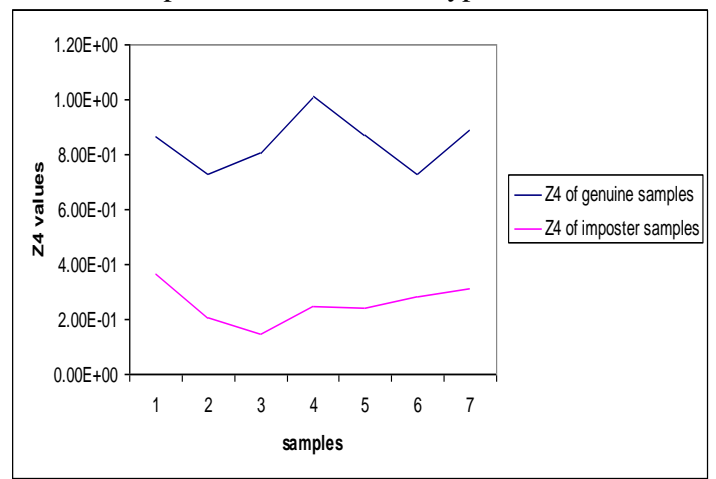

Fig. 6 Plot of z-score of of $h v$ values
The Pearson correlation coefficient was computed. The correlation gives the measure of the linear dependence between two values. The correlation was computed for each consecutive $h v$ values. The correlation $\rho$ for the values $x$ and $y$ is calculated using $\rho_{j}=\operatorname{cov}(x, y) / \sigma_{x} \sigma_{y}$ where $x=h v_{j}, \quad y=h v_{j+1}$ such that $j=\{1, \ldots, 9\}$. The $x$ and $y$ are the $h v$ values of different wavelets in $w t_{\text {order }}$ and $\sigma_{x}, \sigma_{y}$ denotes its standard deviation. The $\operatorname{cov}(x, y)$ indicates the covariance of $\mathrm{x}$ and $\mathrm{y}$ values such that $\operatorname{cov}(x, y)=\sum_{i=1}^{n}\left(x_{i}-\bar{x}\right)\left(y_{i}-\bar{y}\right)$ where $\bar{x}$ and $\bar{y}$ are the mean values. The correlation values for genuine and imposter samples are given in Table 6 .

Table 6. Correlation coefficient values

\begin{tabular}{|c|c|c|c|c|c|c|c|c|c|}
\hline Sample & $\rho_{1}$ & $\rho_{2}$ & $\rho_{3}$ & $\rho_{4}$ & $\rho_{5}$ & $\rho_{6}$ & $\rho_{7}$ & $\rho_{8}$ & $\rho_{9}$ \\
\hline genuine & 0.9 & 0.8 & 0.7 & 0.8 & 0.9 & 0.8 & 0.9 & 0.9 & 0.8 \\
\hline imposter & 0.3 & 0.2 & 0.3 & 0.1 & 0.2 & 0.1 & 0.2 & 0.2 & 0.1 \\
\hline
\end{tabular}

\section{MAGNITUDE BASED APPROACH}

The magnitude of horizontal, vertical and diagonal coefficients are denoted as $h m_{i}, v m_{i}$ and $d m_{i}$ where $i=\{1,2,3,4,5,8,9,12$, $13,14,15\}$. The magnitude values follow a particular sequence of transition. Observing $\mathrm{hm}, \mathrm{vm}$ and $d \mathrm{~m}$ values of any ncseg, the two transition types are learnt. In the first type, the change in magnitude values is from lower to higher and again to lower with reference to $h m, v m$ and $d m$ values respectively. The kind of transition is denoted by P. In the second type, the transition is from higher values in $\mathrm{hm}$ to lower values in the $\mathrm{vm}$ and $\mathrm{dm}$. The kind of transition is denoted by R. Figure 7 show the transitions.

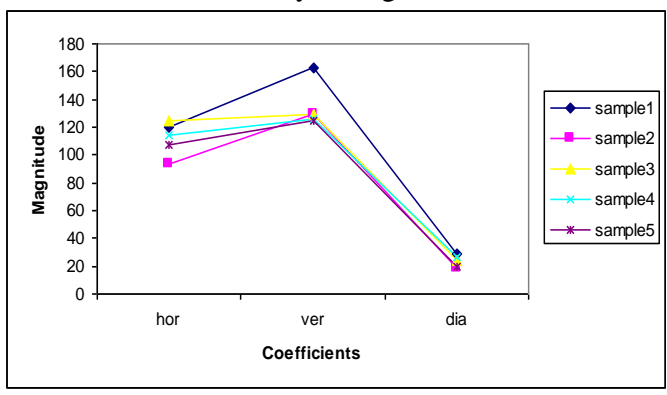

(a)

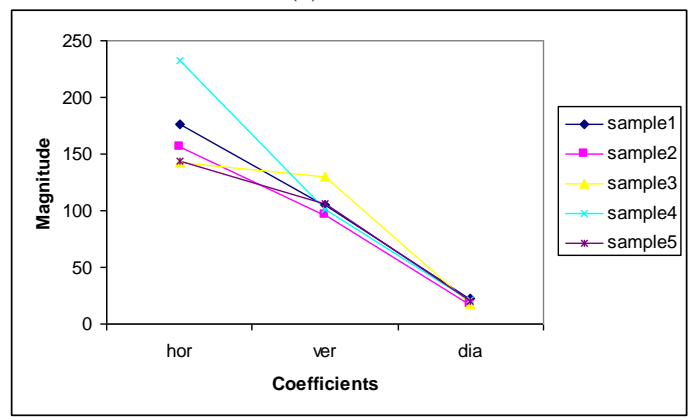

(b)

Fig. 7: (a) $\mathbf{P}$ transition (b) $\mathbf{R}$ transition 


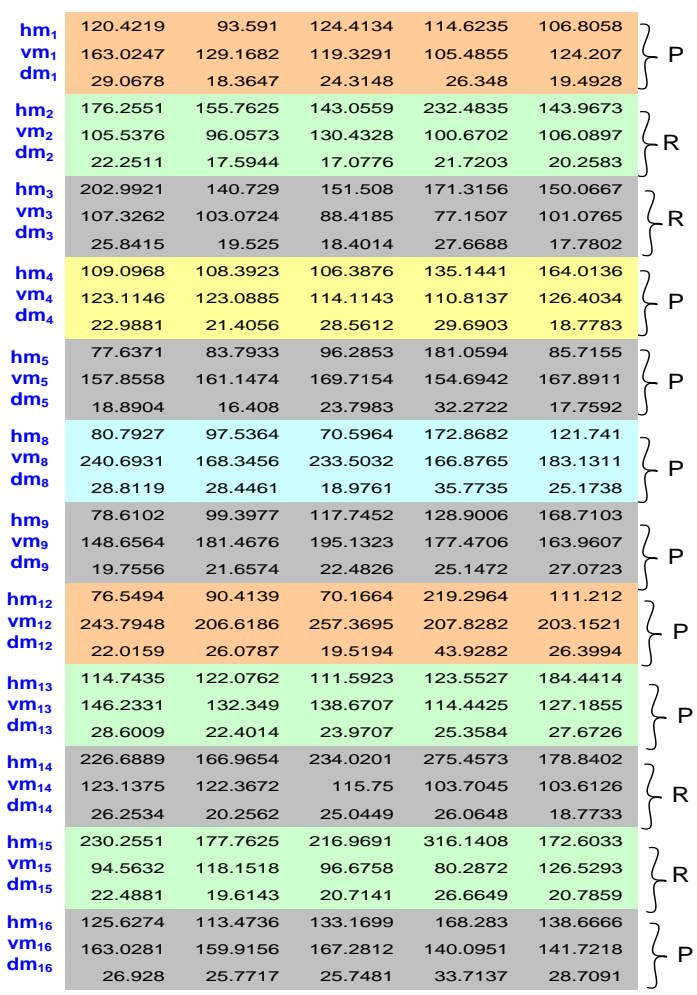

Fig. 8: Formation of a pattern

The sequence of $\mathrm{P}$ and $\mathrm{R}$ follow a particular pattern for a subject. For instance, a pattern $=\{\mathrm{P}, \mathrm{R}, \mathrm{R}, \mathrm{P}, \mathrm{P}, \mathrm{P}, \mathrm{P}, \mathrm{P}, \mathrm{P}, \mathrm{P}, \mathrm{P}, \mathrm{P}, \mathrm{P}\}$. The sequence is in the order considering ncseg $_{i}$ where $i=\{1,2,3$, $4,5,8,9,12,13,14,15,16\}$. The formation of a pattern is shown in Figure 8.

\begin{tabular}{|c|c|c|c|c|c|}
\hline $\mathrm{hm}$ & 120.4219 & 93.591 & 124.4134 & 114.6235 & 106.8058 \\
\hline \multirow{2}{*}{$\begin{array}{l}\mathrm{vm}_{1} \\
\mathrm{dm}_{1}\end{array}$} & 163.0247 & 129.1682 & 119.3291 & 105.4855 & 124.207 \\
\hline & 29.0678 & 18.3647 & 24.3148 & 26.348 & 19.4928 \\
\hline \multirow{3}{*}{$\begin{array}{l}\mathrm{hm}_{2} \\
\mathrm{vm}_{2} \\
\mathrm{dm}_{2}\end{array}$} & 176.2551 & 155.7625 & 143.0559 & 232.4835 & 143.9673 \\
\hline & 105.5376 & 96.0573 & 130.4328 & 100.6702 & 106.0897 \\
\hline & 22.2511 & 17.5944 & 17.0776 & 21.7203 & 20.2583 \\
\hline \multirow{3}{*}{$\begin{array}{l}\mathrm{hm}_{3} \\
\mathrm{vm}_{3} \\
\mathrm{dm}_{3}\end{array}$} & 202.9921 & 140.729 & 151.508 & 171.3156 & 150.0667 \\
\hline & 107.3262 & 103.0724 & 88.4185 & 77.1507 & 101.0765 \\
\hline & 25.8415 & 19.525 & 18.4014 & 27.6688 & 17.7802 \\
\hline \multirow{3}{*}{$\begin{array}{l}\mathrm{hm}_{4} \\
\mathrm{vm}_{4} \\
\mathrm{dm}_{4}\end{array}$} & 109.0968 & 108.3923 & 106.3876 & 135.1441 & 164.0136 \\
\hline & 123.1146 & 123.0885 & 114.1143 & 110.8137 & 126.4034 \\
\hline & 22.9881 & 21.4056 & 28.5612 & 29.6903 & 18.7783 \\
\hline \multirow{3}{*}{$\begin{array}{l}\mathbf{h m}_{13} \\
\mathrm{vm}_{13} \\
\mathrm{dm}_{13}\end{array}$} & 114.7435 & 122.0762 & 111.5923 & 123.5527 & 184.4414 \\
\hline & 146.2331 & 132.349 & 138.6707 & 114.4425 & 127.1855 \\
\hline & 28.6009 & 22.4014 & 23.9707 & 25.3584 & 27.6726 \\
\hline \multirow{3}{*}{$\begin{array}{l}\mathbf{h m}_{14} \\
\mathrm{vm}_{14} \\
\mathrm{dm}_{14}\end{array}$} & 226.6889 & 166.9654 & 234.0201 & 275.4573 & 178.8402 \\
\hline & 123.1375 & 122.3672 & 115.75 & 103.7045 & 103.6126 \\
\hline & 26.2534 & 20.2562 & 25.0449 & 26.0648 & 18.7733 \\
\hline \multirow{3}{*}{$\begin{array}{l}\mathrm{hm}_{15} \\
\mathrm{vm}_{15} \\
\mathrm{dm}_{15}\end{array}$} & 230.2551 & 177.7625 & 216.9691 & 316.1408 & 172.6033 \\
\hline & 94.5632 & 118.1518 & 96.6758 & 80.2872 & 126.5293 \\
\hline & 22.4881 & 19.6143 & 20.7141 & 26.6649 & 20.7859 \\
\hline \multirow{3}{*}{$\begin{array}{l}\mathrm{hm}_{16} \\
\mathrm{vm}_{16} \\
\mathrm{dm}_{16}\end{array}$} & 125.6274 & 113.4736 & 133.1699 & 168.283 & 138.6666 \\
\hline & 163.0281 & 159.9156 & 167.2812 & 140.0951 & 141.7218 \\
\hline & 26.928 & 25.7717 & 25.7481 & 33.7137 & 28.7091 \\
\hline
\end{tabular}

Fig. 9: Formation of a pattern after elimination of segments $\{5,6,8,9\}$
Table 7. Pattern types for subjects in ICE database

\begin{tabular}{|c|c|c|}
\hline Pattern No. & Pattern & Subjects \\
\hline 1 & P R R P P R R P & $3,6,17,24,27,28,51$ \\
\hline 2 & P R R R P R R P & $1,13,16,21,43,44$ \\
\hline 3 & P R R R P R R R & $9,50,57,26,45,87$ \\
\hline 4 & P P R R P R R P & $14,7,47,10,19,78,80$ \\
\hline 5 & P R R R R R R R & 15,22 \\
\hline 6 & R R R R P R R P & $18,34,53,75,56,76,86$ \\
\hline 7 & R R R R R P P P & $32,79,81,83$ \\
\hline 8 & P P P P R R R P & $33,54,38$ \\
\hline 9 & P P P P P R R P & $35,62,4,5,23,39,41$ \\
\hline 10 & P R R P R R R P & $63,49,66$ \\
\hline 11 & P R R P P R R R & $68,12,58,72,23$ \\
\hline 12 & R R R P P R R P & 74,88 \\
\hline 13 & P R P P P R R P & 8,65 \\
\hline 14 & P P P P R R R P & $11,42,67$ \\
\hline 15 & P R R R R R R P & 30,95 \\
\hline 16 & P P R P P R R P & $46,59,89$ \\
\hline 17 & P R P P P R R P & $60,70,82,106$ \\
\hline 18 & P R R P R R R R & $77,61,2$ \\
\hline 19 & R R R P R R R P & $85,29,37,48$ \\
\hline 20 & P R R P P P P P & $52,55,64,69,71$ \\
\hline 21 & R R R P P P P P & $25,31,40$ \\
\hline 22 & R P R P R R R R & $20,36,84$ \\
\hline
\end{tabular}

In segments, ncseg $_{5}$, ncseg $_{8}$, ncseg $_{9}$ and $n c s e g_{12}$, the transitions are only of type $\mathrm{P}$ in all samples. The variations in the segments are negligible. Eliminating the segments in the sequence, the generation of a pattern is shown in Figure 9. The listing of patterns and subjects are given in Table 7.

The $h m, v m$ and $d m$ values are segregated as shown in Figure 10. The minimum average and maximum average values are computed using the averages of each row. The minimum average segments, minavg $_{\text {seg }}$ and maximum average segments, maxavg ${ }_{\text {seg }}$ are determined for each of the $h m, v m$ and $d m$ values. For instance, minavg $_{\text {seg }}=\left\{h m_{1}, v m_{3}, d m_{2}\right\}$ and maxavg $_{\text {seg }}=\left\{h m_{15}, v m_{16}\right.$, $\left.d m_{16}\right\}$ as shown in Figure 11. 

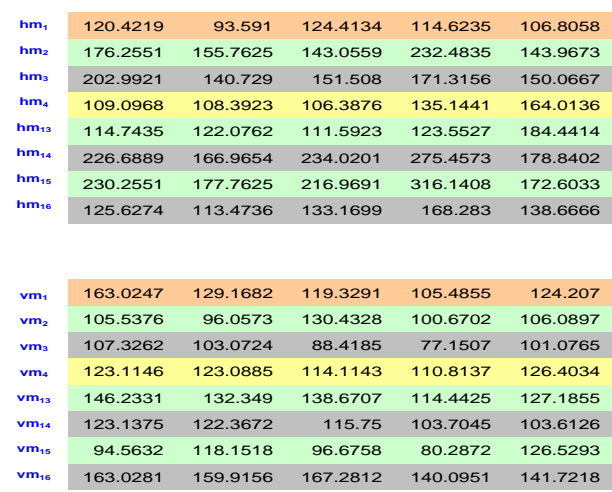

\begin{tabular}{l|rrrrr|}
$\mathbf{d m}_{1}$ & 29.0678 & 18.3647 & 24.3148 & 26.348 & 19.4928 \\
\hline $\mathbf{d m}_{2}$ & 22.2511 & 17.5944 & 17.0776 & 21.7203 & 20.2583 \\
$\mathbf{d m}_{3}$ & 25.8415 & 19.525 & 18.4014 & 27.6688 & 17.7802 \\
\hline $\mathbf{d m}_{4}$ & 22.9881 & 21.4056 & 28.5612 & 29.6903 & 18.7783 \\
$\mathbf{d m}_{13}$ & 28.6009 & 22.4014 & 23.9707 & 25.3584 & 27.6726 \\
\hline $\mathbf{d m}_{14}$ & 26.2534 & 20.2562 & 25.0449 & 26.0648 & 18.7733 \\
\hline $\mathbf{d m}_{15}$ & 22.4881 & 19.6143 & 20.7141 & 26.6649 & 20.7859 \\
\hline $\mathbf{d m}_{16}$ & 26.928 & 25.7717 & 25.7481 & 33.7137 & 28.7091 \\
\hline
\end{tabular}

Fig. 10: Separation of $\mathrm{hm}, \mathrm{vm}$ and $\mathrm{dm}$ values for a pattern
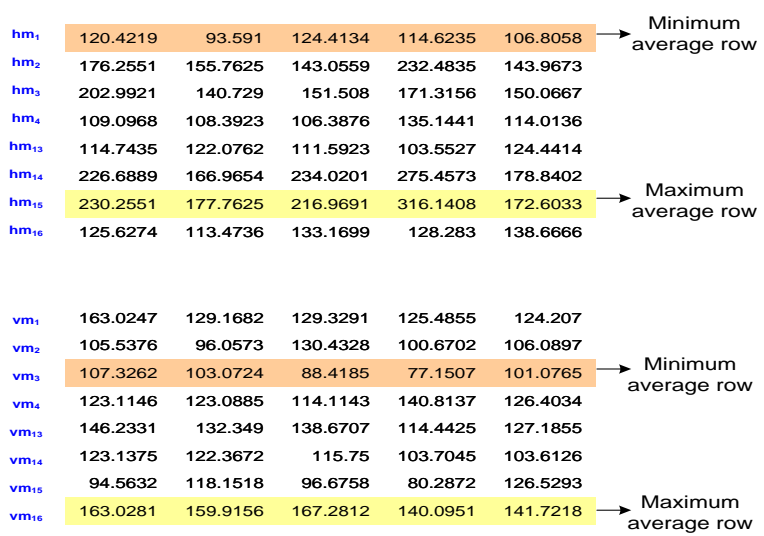

$\begin{array}{lrrrrrl}\mathrm{dm}_{1} & 29.0678 & 18.3647 & 24.3148 & 26.348 & 19.4928 & \\ \mathrm{dm}_{2} & 22.2511 & 17.5944 & 17.0776 & 21.7203 & 20.2583 & \rightarrow \text { Minimum } \\ \mathrm{dm}_{3} & 25.8415 & 19.525 & 18.4014 & 27.6688 & 17.7802 & \\ \mathrm{dm}_{4} & 22.9881 & 21.4056 & 28.5612 & 29.6903 & 18.7783 & \\ \mathrm{dm}_{13} & 28.6009 & 22.4014 & 23.9707 & 25.3584 & 27.6726 & \\ \mathrm{dm}_{14} & 26.2534 & 20.2562 & 25.0449 & 26.0648 & 18.7733 & \\ \mathrm{dm}_{15} & 22.4881 & 19.6143 & 20.7141 & 26.6649 & 20.7859 & \\ \mathrm{dm}_{16} & 26.928 & 25.7717 & 25.7481 & 33.7137 & 28.7091 & \rightarrow \text { Maximum } \\ & & & & & & \end{array}$

Fig. 11 Minimum and maximum average values for a pattern

\section{EXPERIMENTAL RESULTS}

Experiments are conducted on ICE database [18]. The database consists of 89 subjects with 7 samples per subject. The wavelet based recognition use magnitude values of the coefficients. A unique code is generated for each subject. The code recognizes a person from others in the database. minavg $_{\text {seg }}$ and maxavg $g_{\text {seg }}$ values represent the code for an individual. The recognition rate is $100 \%$. Table 8 depicts minavg $_{\text {seg }}$ and maxavg $_{\text {seg }}$ values for first and second level of decomposition. The sequence indicates $\mathrm{hm}$, $v m$ and $d m$ values of minavg $g_{\text {seg }}$ and maxavg $_{\text {seg }}$. For instance, level 1 entry for subject 1 is $\{h m 1, v m 2, d m 3, h m 15, v m 16, d m 16\}$. The first three values $\{h m 1, v m 2, d m 3\}$ correspond to minavg $g_{\text {seg. }}$. The next three values $\{h m 15, v m 16, d m 16\}$ correspond to maxavg ${ }_{\text {seg }}$ values. The values are unique for each person in the database.
Table 8. minavg $_{\text {seg }}$ and maxavg seg $_{\text {values for first and second }}$ level wavelet decomposition for ICE database

\begin{tabular}{|c|c|c|}
\hline $\begin{array}{l}\text { Subjec } \\
\mathrm{t}\end{array}$ & Level 1 decomposition & Level 2 decomposition \\
\hline 1 & $\begin{array}{c}\mathrm{hm}_{1}, \mathrm{vm}_{2}, \mathrm{dm}_{3}, \mathrm{hm}_{15}, \\
\mathrm{vm}_{16}, \mathrm{dm}_{16}\end{array}$ & $\begin{array}{c}\mathrm{hm}_{13}, \mathrm{vm}_{1}, \mathrm{dm}_{3}, \mathrm{hm}_{3}, \mathrm{vm}_{2}, \\
\mathrm{dm}_{1}\end{array}$ \\
\hline 2 & $\begin{array}{c}\mathrm{hm}_{4}, \mathrm{vm}_{15}, \mathrm{dm}_{3}, \mathrm{hm}_{15}, \\
\mathrm{vm}_{1}, \mathrm{dm}_{1}\end{array}$ & $\begin{array}{c}\mathrm{hm}_{3}, \mathrm{vm}_{15}, \mathrm{dm}_{4}, \mathrm{hm}_{15}, \mathrm{vm}_{2}, \\
\mathrm{dm}_{1}\end{array}$ \\
\hline 3 & $\begin{array}{c}\mathrm{hm}_{1}, \mathrm{vm}_{14}, \mathrm{dm}_{16}, \mathrm{hm}_{15}, \\
\mathrm{vm}_{1}, \mathrm{dm}_{13}\end{array}$ & $\begin{array}{c}\mathrm{hm}_{1}, \mathrm{vm}_{15}, \mathrm{dm}_{3}, \mathrm{hm}_{14}, \mathrm{vm}_{1}, \\
\mathrm{dm}_{13}\end{array}$ \\
\hline 4 & $\begin{array}{c}\mathrm{hm}_{2}, \mathrm{vm}_{4}, \mathrm{dm}_{13}, \mathrm{hm}_{1}, \\
\mathrm{vm}_{16}, \mathrm{dm}_{13}\end{array}$ & $\begin{array}{c}\mathrm{hm}_{4}, \mathrm{vm}_{4}, \mathrm{dm}_{2}, \mathrm{hm}_{13}, \mathrm{vm}_{16}, \\
\mathrm{dm}_{13}\end{array}$ \\
\hline - & · & $\cdot$ \\
\hline$\cdot$ & $\cdot$ & $\cdot$ \\
\hline 88 & $\begin{array}{c}\mathrm{hm}_{4}, \mathrm{vm}_{15}, \mathrm{dm}_{3}, \mathrm{hm}_{1}, \\
\mathrm{vm}_{13}, \mathrm{dm}_{13}\end{array}$ & $\frac{\mathrm{hm}_{1}, \mathrm{vm}_{2}, \mathrm{dm}_{3}, \mathrm{hm}_{4}, \mathrm{vm}_{13},}{\mathrm{dm}_{15}}$ \\
\hline 89 & $\begin{array}{c}\mathrm{hm}_{2}, \mathrm{vm}_{4}, \mathrm{dm}_{13}, \mathrm{hm}_{14}, \\
\mathrm{vm}_{4}, \mathrm{dm}_{2}\end{array}$ & $\begin{array}{c}\mathrm{hm}_{14}, \mathrm{vm}_{15}, \mathrm{dm}_{2}, \mathrm{hm}_{1}, \mathrm{vm}_{3}, \\
\mathrm{dm}_{4}\end{array}$ \\
\hline
\end{tabular}

\section{CONCLUSION}

Wavelet transformation is applied for the non-core components. The non-core components are the regions which show variations in the texture and intensity values. The summation and magnitude values of the detailed coefficients form a specific representation for recognition. The summation based method implements different type of wavelets, Haar, db4, symlets, coiflets and biorthogonal. The results obtained using db4 are promising for recognition. The magnitude of detail coefficients is the basis for generation of unique code. The identification of unique code for each person is the novel recognition process.

\section{ACKNOWLEGMENTS}

The authors would like to thank Patrick J Flynn, Professor, Department of Computer Science and Engineering, University of Notre Dame, USA for providing ICE database.

\section{REFERENCES}

[1] Rafeal C. Gonzalez and Richard E. Woods. 2005. Digital Image Processing, $2^{\text {nd }}$ Edition, Pearson Education.

[2] C.A.Bouman. 2011. Connected Component Analysis, Digital Image Processing, https://engineering.purdue.edu/bouman/ ece637/notes/pdf/ConnectComp.pdf.

[3] Abeer George Ghuneim. 2000. Defining Connectivity, http://www.imageprocessingplace.com.

[4] Earl Gose, Richard Johnsonbaugh and Steve Jost. 2006. Pattern Recognition and Image Analysis, 1st Edition, Prentice-Hall of India Private Limited.

[5] Robi Polikar. 1999. The Wavelet Tutorial. 
[6] Amara Graps. 1995. An Introduction to Wavelets, Computing in Science and Engineering, vol. 2, no. 2, pp. 50-61.

[7] Mohammed A. Salem, Nivin Ghamry and Beate Meffert. 2009. Daubechies Versus Biorthogonal Wavelets for Moving Object Detection in Traffic Monitoring Systems,

http://www2.informatik.hu-berlin.de/sam/preprint/m.

[8] Junxing Ma, Jijun Xue, Shengjun Yang and Zhengjia He. 2003. A study of the construction and application of a Daubechies wavelet-based beam element, Finite Elements in Analysis and Design, vol. 39, pp. 965-975.

[9] J. Daugman. 1993. High Confidence Visual Recognition by a Test of Statistical Independence, IEEE Trans. Pattern Analysis and Machine Intelligence, vol. 15, No.11, pp.11481161 .

[10] Karen Hollingsworth, Sarah Baker, Sarah Ring, Kevin W. Bowyer and Patrick J.Flynn. 2009. Recent Research Results In Iris Biometrics, SPIE 7306B: Biometric Technology for Human Identification VI.

[11] R. Wildes, J. Asmuth, G. Green, S. Hsu, R. Kolczynski, J. Matey, and S. McBride. 1996. A machine-vision system for iris recognition, Mach. Vis. Applic., vol. 9, pp. 1-8.

[12] Li Ma, Tieniu Tan, Yunhong Wang, Dexin Zhang. 2003. Personal Identification based on Iris Texture Analysis, IEEE Transactions on Pattern Analysis and Machine Intelligence, Vol.25, No.12, pp. 1519-1533.
[13] Zhenan Sun, Yunhong Wang, Tieniu Tan and Jiali Cui, 2004. Improving Iris Recognition Accuracy Via Cascaded Classifiers, Biometric Authentication. LNCS 3072, pp. 1-11.

[14] H. Proena and L.A. Alexandre. 2006. Iris Segmentation Methodology for Noncooperative Iris Recognition. IEE Proc. Vision, Image, and Signal Processing, vol. 153, no. 2, pp. 199-205

[15] Aditya Abhyankar and Stephanie Schuckers. 2010. Novel Biorthogonal Wavelet based Iris Recognition for Robust Biometric System, International Journal of Computer Theory and Engineering, vol. 2, No. 2, pp. 1793-8201.

[16]Agus Harjoko, Sri Hartati and Henry Dwiyasa. 2009. A Method for Iris Recognition Based on 1D Coiflet Wavelet.World Academy of Science, Engineering and Technology, vol. 56, pp. 1-4.

[17] Zhengmao Ye, Yongmao Ye, Hang Yin, Habib Mohamadian. 2009. Integration of Wavelet Fusion and Adaptive Contrast Stretching for Object Recognition with Quantitative Information Assessment, ICGST-GVIP Journal, vol. 8, Issue V, pp. 33-42.

[18]Xiaomei Liu, Bowyer K.W. and Flynn P.J. 2005. Experimental Evaluation of Iris Recognition, IEEE Computer Society Conference on Computer Vision and Pattern Recognition Workshops, pp. 158-165. 